\title{
Chronischer Schmerz: Unterschätzt und unterbehandelt
}

\author{
Die Versorgungssituation in Österreich ist verbesserungswürdig.
}

Lange anhaltend, häufig die Notwendigkeit einer multimodalen Therapie und Schmerzlinderung als Ziel - das sind die Eckpunkte des chronischen Schmerzes. Allerdings ist das Phänomen chronischer Schmerz in Österreich weiterhin unterschätzt und unterbehandelt. Die Lebensqualität der Betroffenen ist dadurch massiv eingeschränkt und der Volkswirtschaft entsteht jährlich ein Schaden in Millionenhöhe. Ein von Joanneum Research erstellter Forschungsbericht zur Versorgungssituation bei Schmerz in Österreich wurde Anfang Dezember in Wien präsentiert. Immerhin sind etwa 20 Prozent der Bevölkerung von chronischen Schmerzen betroffen, wovon etwa 6,25 Prozent eine dauernde ärztliche Versorgung wegen der Schwere ihrer Schmerzen benötigen. Quer durch alle Altersgruppen.

Die Bestandsaufnahme zeigt Handlungsbedarf: Schmerz ist die zweithäufigste Ursache für einen Arztbesuch, 39 Prozent der Patienten suchen mindestens einmal wöchentlich den Arzt wegen ihrer Beschwerden auf und bis der Patient die richtige Diagnose erhält, vergehen 1,7 bis 2,3 Jahre. Auch an den Spitalsaufenthalten haben Schmerzpatienten einen nicht unerheblichen Anteil: 1,5 Prozent der Spitalsentlassungen weisen als Diagnose Schmerz auf, wobei die durchschnittliche Aufenthaltsdauer 7,3 Tage beträgt. Schmerzambulanzen in Österreich bieten ein breites multimodales Behandlungsangebot, berichtete Louise Jane Schmidt von Joanneum Research: „Es gibt interdisziplinäre Besprechungen, Fortbildung und in jeder Ambulanz einen Facharzt mit Schmerzdiplom. Allerdings arbeitet nur etwa die Hälfte der Schmerzambulanzen mit standardisierten Schmerzfragebögen und zwei Drittel halten sich an Leitlinien." Verbesserungspotential bestehe darüberhinaus in der $\mathrm{Zu}$ sammenarbeit mit dem niedergelassenen Bereich.

\section{Interdisziplinäre Zusammenarbeit und abgestufte Versorgung}

Die Wichtigkeit der interdisziplinären Zusammenarbeit und die verstärkte Förderung und Strukturierung der schmerztherapeutischen Ausbildung unterstrich

auch Priv.-Doz. Dr. Christian Lampl, der kommende Präsident der Österreichischen Schmerzgesellschaft und Leiter der Schmerzklinik des Krankenhauses der Barmherzigen Schwestern in Linz. „Weit mehr als 90 Prozent der Schmerzkranken könnten ambulant behandelt werden." Derzeit sei man jedoch von einer sinnvoll abgestuften Versorgung durch den Hausarzt, den

\section{Wir brauchen den konsequenten Einsatz multimodaler Therapiekonzepte, in denen Medikamente, Methoden der Medizintechnik, Physikalische Medizin, Psychotherapie und geeignete Methoden der Komplementärmedizin individuell kombiniert werden.}

Schmerzarzt aus den Fächern Neurologie, Anästhesie oder ähnlichen bis zur interdisziplinären Schmerzambulanz oder Schmerzabteilung noch weit entfernt. „Wir brauchen“, so Lampl, „den konsequenten Einsatz multimodaler Therapiekonzepte, in denen Medikamente, Methoden der Medizintechnik, Physikalische Medizin, Psychotherapie und geeignete Methoden der Komplementärmedizin individuell kombiniert werden, um für Patienten ein Optimum an Schmerzlinderung zu bewirken." Eine wichtige Zusammenarbeit sei in Zukunft auch jene mit Patientenorganisationen. Denn das Hauptproblem chronischen Schmerzes ist die Beeinträchtigung der Lebensqualität des Betroffenen. Und: Schmerz ist die größte Sorge des Patienten.

Chronische Schmerzen sind auch für einen wesentlichen Anteil an Krankenständen verantwortlich, stellte der Sozialmediziner Univ.-Prof. Dr. Bernhard Schwarz von der Universität Wien fest. Schmerz sei das Leitsymptom bei vielen relevanten Diagnosen, aber er gilt derzeit nicht als eigenes Krankheitsbild. Daher ist eine statistische Erfassung relativ schwierig und die Datenlage in Österreich schlecht. Gerade bei chronischen Schmerzen komme auch dem Patienten eine wesentliche Rolle zu: „Schmerzprävention ist in vielen Fällen eine Frage des Lebensstils." Allerdings ist dies in Österreich auch eine kulturelle Frage. Denn immer noch ist Österreich bezüglich der Ausgaben im Bereich der Reparaturmedizin führend, hinsichtlich der Ausgaben für Gesundheitsvorsorge jedoch unter dem EU-Durchschnitt. Die Rolle der Eigenverantwortung betonte auch Dr. Winfried Koller von der Steirischen Akademie für Allgemeinmedizin: „Der Patient sollte mehr in die Pflicht genommen werden,“ Dies sei auch eine Frage der Politik, die es bisher nicht geschafft habe, den Patienten durch die Vorsorge zu lenken. Der diesbezügliche Vorstoß der Sozialversicherung

der gewerblichen Wirtschaft möchte genau diese Richtungsänderung bewirken. Mit Zielvereinbarungen, die der Hausarzt mit dem Patienten trifft, soll dieser motiviert werden, für seine Gesundheit aktiv zu werden - und erhält dadurch nicht nur eine bessere Gesundheit sondern auch einen finanziellen Anreiz von seiner Krankenkasse.

\section{Konkrete Empfehlungen für bessere Schmerzversorgung}

Die Empfehlungen zur Verbesserung der Schmerzversorgung reichen von der Vermeidung einer drohenden Chronifizierung und einer frühzeitigen Risikoreduktion über die Nutzung standardisierter Dokumentationen wie Checkliste, Behandlungspfade $u$.ä. sowie die Orientierung an Evidenz-basierten Leitlinien bis zur abgestuften Versorgung, der Verbesserung der Zusammenarbeit und der Einbindung von Psycho- und Physiotherapeuten in das Kernteam sowie die regelmäßige Teilnahme an Schmerzkonferenzen und Fortbildungen.

Quelle: PK Joanneum Research, Wien 7.12. 2011

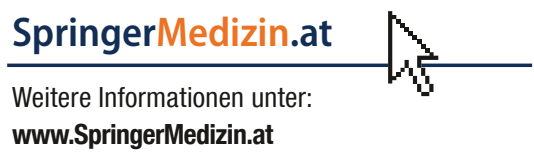

